\title{
MOD 2 SEIBERG-WITTEN INVARIANTS OF HOMOLOGY TORI
}

\author{
Daniel Ruberman and Sašo Strle
}

\section{Introduction}

While the Seiberg-Witten equations are defined for any Spin ${ }^{\mathrm{c}}$ structure on a smooth 4-manifold, there is particular interest in the Seiberg-Witten equations associated to a spin structure. One reason for this is that the 4-dimensional spin representation has a quaternionic structure, which gives rise to a large symmetry group of the 'trivial' reducible solution to the Seiberg-Witten equations. This group, denoted $J$, is generated by $\mathrm{U}(1)$ and the quaternion $j$. The interplay of this symmetry group and the deformation theory of the trivial solution is central to Furuta's proof of the ' $10 / 8$ ' inequality, which constrains the homotopy type of smooth spin manifolds. A closely related argument (known to Kronheimer and Furuta as well) was used by Morgan-Szabó [12] to determine the mod 2 Seiberg-Witten invariant of homotopy K3 surfaces and other simply-connected spin manifolds.

In this paper we show that the mod 2 Seiberg-Witten invariant can be determined for a spin manifold $X$ which has the same homology groups as the 4-torus $T^{4}$. The value depends on the structure of the cohomology ring of $X$, and in particular on the 4 -fold cup product $\Lambda^{4} H^{1}(X) \rightarrow H^{4}(X)$. For the rest of the paper, $X$ will denote a (spin) homology torus, by which we mean an oriented spin 4-manifold with $H_{1}(X ; \mathbf{Z}) \cong \mathbf{Z}^{4}$ and $H_{2}(X ; \mathbf{Z}) \cong \mathbf{Z}^{6}$. The cup product on $H^{2}(X)$ is readily seen to be hyperbolic, but the cup product on $H^{1}(X)$ is not determined by the dimensions of these groups. Let us define $\operatorname{det}(X)$, the determinant of $X$, to be the absolute value of

$$
<\alpha_{1} \cup \alpha_{2} \cup \alpha_{3} \cup \alpha_{4},[X]>,
$$

where $\left\{\alpha_{j}\right\}$ is a basis for $H^{1}(X ; \mathbf{Z})$.

Theorem A. The value of the Seiberg-Witten invariant for the Spin ${ }^{\mathrm{c}}$ structure on $X$ with trivial determinant line is congruent $(\bmod 2)$ to the determinant of $X$.

Received March 9, 2000.

The first author was partially supported by NSF Grant 4-50645. The second author was supported in part by the Ministry for Science and Technology of the Republic of Slovenia Grant No. J1-0885-0101-98. 
Let $X$ be a homology torus, and let $W \rightarrow X$ be a $\operatorname{Spin}^{\mathrm{c}}$ bundle with trivial determinant line $L \rightarrow X$. We fix a square root $L^{1 / 2}$ of the determinant bundle, or equivalently a spin structure on $X$. A trivialization of $L^{1 / 2}$ provides us with a preferred origin in the space $\mathcal{A}$ of $U(1)$-connections on $L^{1 / 2}$, namely the (smooth) product connection $A_{0}$. Note that although there are many spin structures on $X$, they are all isomorphic as $\operatorname{Spin}^{\mathrm{c}}$ structures; the choice of spin structure is reflected only in the (gauge equivalence class of the) above mentioned trivialization and hence in the choice of $A_{0}$, but does not affect the argument.

Recall that the configuration space for the Seiberg-Witten equations is $\mathcal{C}=$ $\mathcal{A} \oplus \Gamma\left(W^{+}\right)$; however, we restrict the equations to the slice $\mathcal{C}^{\prime}=\mathcal{K} \oplus \Gamma\left(W^{+}\right)$ where $\mathcal{K}=\left\{A \in \mathcal{A} \mid d^{*}\left(A-A_{0}\right)=0\right\}$. With this restriction, the moduli space $\mathcal{M}$ of solutions to the Seiberg-Witten equations is the quotient of the space of solutions by the action of the group of harmonic gauge transformations; denote by $\mathcal{G}_{0}$ the based gauge group of harmonic gauge transformations. The formal dimension of the moduli space $\mathcal{M}$ for the trivial $\mathrm{Spin}^{\mathrm{c}}$ structure on $X$ is zero (as is the index of Dirac operator $D_{A}: \Gamma\left(W^{+}\right) \rightarrow \Gamma\left(W^{-}\right)$for any $\operatorname{Spin}^{\mathrm{c}}$ connection $\nabla_{A}$ on $\left.W\right)$. In the absence of any perturbation terms, however, the moduli space is not cut out transversally because it contains the 'dual' 4 -torus $T^{*} \cong H^{1}\left(X ; S^{1}\right)$ of reducible solutions $[A, 0]$ with $A$ harmonic. This dual torus is covered (under the action of $\left.\mathcal{G}_{0}\right)$ by the space of harmonic connections $\widetilde{T}^{*}=A_{0}+i \mathcal{H}^{1}(X) \subset \mathcal{K}$. In the case $X=T^{4}$ the dual torus coincides with the moduli space; moreover, the only point along $T^{*}$ where the Dirac operator has nontrivial kernel is $\left[A_{0}, 0\right]$. From this, and the structure of the quadratic term of the equations, one can show that the Seiberg-Witten invariant (for the trivial Spin $^{\mathrm{c}}$ structure) of $T^{4}$ is \pm 1 ; this of course implies Theorem A for $X=T^{4}$. To prove it in the general case, we perturb the equations along $\widetilde{T}^{*}$ so that they become as nondegenerate as possible; this is done in two stages - we deal with the linear perturbations in section 2 , and with nonlinear ones in section 3 . The invariant is then determined by the count of solutions which lie near $T^{*}$; as in [12], we use the involution $j$ on the moduli space of solutions, induced by taking the dual Spin ${ }^{\mathrm{c}}$ structure, to pair off the solutions away from $T^{*}$.

The spaces of sections are $L_{2}^{2}$ unless stated otherwise; in particular, this holds for the configuration space $\mathcal{C}^{\prime}$. The gauge transformations are in the space $L_{3}^{2}$.

\section{Dirac operators along $T^{*}$}

Recall that the Seiberg-Witten equations are given by a map $S W: \mathcal{K} \oplus$ $\Gamma\left(W^{+}\right) \rightarrow i \Omega_{+}^{2}(X) \oplus \Gamma\left(W^{-}\right),(A, \psi) \mapsto\left(F_{A}^{+}-q(\psi), D_{A} \psi\right)$, where $F_{A}^{+}$is the self-dual part of the curvature and $q$ is a quadratic map. Thus for any $A \in \widetilde{T}^{*}$, the linearization of the equations at $(A, 0)$ is $\left(d^{+}, D_{A}\right)$. Since $d^{+}$does not depend on $A$, the behavior of the linearizations of the Seiberg-Witten equations along $\widetilde{T}^{*}$ is described by the family of Dirac operators $\left\{D_{A}, A \in \widetilde{T}^{*}\right\}$. We think of this family as a morphism of trivial bundles $\widetilde{T}^{*} \times \Gamma\left(W^{+}\right) \rightarrow \widetilde{T}^{*} \times \Gamma\left(W^{-}\right)$. Note that the gauge group $\mathcal{G}_{0}$ acts freely on the base space $\widetilde{T}^{*}$ of these bundles, 
so dividing out by the action of $\mathcal{G}_{0}$ produces bundles $\boldsymbol{\Gamma}\left(W^{ \pm}\right) \rightarrow T^{*}$ with fibres $\Gamma\left(W^{ \pm}\right)$. Each of these bundles supports a free $J$-action which is compatible with the $J$-action on the base $T^{*}$; we call any such bundle a $J$-bundle. The family of Dirac operators defines a family of Fredholm operators parametrized by $T^{*}$; we denote the resulting morphism by $\boldsymbol{D}: \boldsymbol{\Gamma}\left(W^{+}\right) \rightarrow \boldsymbol{\Gamma}\left(W^{-}\right)$. From above we know that the pointwise index of $\boldsymbol{D}$ is 0 , but the index bundle $\operatorname{Ind}(\boldsymbol{D})$ of $\boldsymbol{D}$ may be nontrivial. We will see that the latter is determined by the cup product on $H^{1}(X)$. It is, therefore, the index computation that links the cup product structure to the behavior of the linear part of the Seiberg-Witten equations along $\widetilde{T}^{*}$.

The calculation of the family index of $\boldsymbol{D}$ is similar to the one arising in the proof $[8,13]$ of the wall-crossing formula for 4 -manifolds with $b^{1}>0$. The Chern character of the index bundle is given by $\operatorname{ch}(\operatorname{Ind}(\boldsymbol{D}))=\operatorname{ch}(\mathbf{L}) /[X]$, since the $\hat{A}$-genus of $X$ is 1 . Here $\mathbf{L} \rightarrow X \times T^{*}$ is the universal line bundle equipped with a connection $\mathbf{A}$ as follows. Let $\alpha_{1}, \ldots, \alpha_{4} \in \mathcal{H}^{1}(X ; \mathbf{Z})$ be a basis and let $t_{k} \mapsto 2 \pi i t_{k} \alpha_{k}$ be coordinates on $T^{*} \cong \mathcal{H}^{1}(X ; i \mathbf{R}) / \mathcal{H}^{1}(X ; 2 \pi i \mathbf{Z})$. The connection 1 -form of $\mathbf{A}$ is given by

$$
2 \pi i \sum_{k} t_{k} \alpha_{k}
$$

By Chern-Weil theory, the first Chern class of $\mathbf{L}$ is then represented by the 2 -form

$$
\Omega=\sum_{k} \alpha_{k} \wedge d t_{k}
$$

and therefore

$$
\operatorname{ch}(\mathbf{L})=1+\Omega+\frac{1}{2} \Omega^{2}+\frac{1}{6} \Omega^{3}+\frac{1}{24} \Omega^{4} .
$$

It is at this point that the cup product structure of $X$ shows up; the formula for the Chern character of the index bundle gives

$$
\operatorname{ch}(\operatorname{Ind}(\boldsymbol{D}))= \pm r\left[\operatorname{vol}_{T^{*}}\right]
$$

where $r$ denotes the determinant of $X$. Consequently, $c_{2}(\operatorname{Ind}(\boldsymbol{D}))= \pm r$ and $c_{1}(\operatorname{Ind}(\boldsymbol{D}))=0$; this suggests that there is a simple model for the index bundle and the construction of this model occupies the rest of the section.

In the proposition below we construct a generic model for the index bundle, realized by stabilizing the domain and the range of the operators. This corresponds to a stabilization of the Seiberg-Witten equations; we will define the stabilized equations via a map

$$
\overline{S W}: \mathcal{K} \oplus \Gamma\left(W^{+}\right) \oplus \mathbf{H}^{n} \rightarrow i \Omega_{+}^{2}(X) \oplus \Gamma\left(W^{-}\right) \oplus \mathbf{H}^{n} .
$$

Proposition 2.1. Suppose that $\operatorname{det}(X)=r$. Then there is a $J \times \mathcal{G}_{0}$-equivariant stabilization of the Seiberg-Witten equations, with reducible solutions along $T^{*}$, such that the corresponding family of Dirac operators has nontrivial kernel at exactly $r$ points on $T^{*}$. 
Proof. As an element of the ordinary $K$-theory of $T^{*}$, the index bundle may be represented as a difference of complex vector bundles. We need to represent $\operatorname{Ind}(\boldsymbol{D})$ as the difference of two genuine $J$-bundles over $T^{*}$, and so adapt the standard argument to the context of $J$-equivariant $K$-theory (compare $[1,5]$ ) as follows. By standard arguments there exists a $\mathbf{C}$-linear morphism $\mathbf{G}_{0}: T^{*} \times$ $\mathbf{C}^{n} \rightarrow \boldsymbol{\Gamma}\left(W^{-}\right)$which is onto the cokernel of $\boldsymbol{D}$. This morphism extends to a $J$-equivariant morphism $\mathbf{G}: T^{*} \times \mathbf{H}^{n} \rightarrow \boldsymbol{\Gamma}\left(W^{-}\right)$, where the product bundle $T^{*} \times \mathbf{H}^{n}$ has the product action of $j$; the $j$-action on the space of quaternions $\mathbf{H}=\mathbf{C} \oplus j \mathbf{C}$ is via right quaternionic multiplication. Given any $[A, 0] \in T^{*}$ and $w \in \mathbf{C}^{n}$ set

$$
\mathbf{G}([A, 0], j w):=j \cdot \mathbf{G}_{0}([j(A), 0], \bar{w}),
$$

and extend by linearity. Perturbing the family of Dirac operators $\boldsymbol{D}$ by the morphism $\mathbf{G}$ produces a $J$-equivariant epimorphism

$$
\begin{aligned}
\overline{\boldsymbol{D}}: \boldsymbol{\Gamma}\left(W^{+}\right) \oplus T^{*} \times \mathbf{H}^{n} & \rightarrow \boldsymbol{\Gamma}\left(W^{-}\right) \\
([A, 0], \psi, w) & \mapsto \boldsymbol{D}_{[A, 0]} \psi+\mathbf{G}([A, 0], w) .
\end{aligned}
$$

Through this we have represented $\operatorname{Ind}(\boldsymbol{D})$ in $J$-equivariant K-theory as the difference of the kernel bundle of $\overline{\boldsymbol{D}}$ and the product $J$-bundle $T^{*} \times \mathbf{H}^{n}$.

Considered as a complex bundle, the kernel bundle $K:=\operatorname{ker} \overline{\boldsymbol{D}}$ splits as a sum $K=K^{\prime} \oplus K^{\prime \prime}$, for dimensional reasons, where $K^{\prime}$ is a trivial complex bundle, and $K^{\prime \prime}$ is a $\mathbf{C}^{2}$-bundle with $c_{2}\left(K^{\prime \prime}\right)= \pm r$ and $c_{1}\left(K^{\prime \prime}\right)=0$. In fact, $K$ splits in the category of $J$-bundles over $T^{*}$, in such a way that $K^{\prime}$ is a trivial $\mathbf{H}^{n-1}$ bundle. To construct this splitting note that any vector bundle over $T^{*}$ with fibre dimension greater than 4 (over $\mathbf{R}$ ) admits a nowhere vanishing section $s$. On any $J$-bundle $M \rightarrow T^{*}$ such a section $s$ gives rise to a trivial $J$-invariant subbundle $N \rightarrow T^{*}$ of complex rank 2 , spanned by $s$ and $\bar{s}([A, 0])=j \cdot s([j(A), 0])$. Moreover, $N$ has a $J$-invariant complement in $M$; the latter can be taken to be perpendicular to $N$ with respect to some (compatible) hermitian inner product on $M$. For the case at hand we choose the standard hermitian structure on $T^{*} \times \mathbf{H}^{n}$ and the $L^{2}$-inner product on the fibres of $\boldsymbol{\Gamma}\left(W^{+}\right)$. Denote the resulting $J$-equivariant isomorphism by $\mathbf{F}^{\prime}: K^{\prime} \rightarrow T^{*} \times \mathbf{H}^{n-1}$.

The bundle $K^{\prime \prime}$ admits a structure of a quaternionic line bundle; we use this to construct a $J$-equivariant morphism $\mathbf{F}^{\prime \prime}: K^{\prime \prime} \rightarrow T^{*} \times \mathbf{H}$, injective everywhere except at $r$ chosen points on $T^{*}$. Let $\mathcal{R} \subset T^{*}$ be a $j$-invariant subset with $r$ elements; such exists for any $r$ since the $j$-action on $T^{*}$ has fixed points (for example $\left.\left[A_{0}, 0\right]\right)$. We choose a section $s_{0}$ of the bundle $K^{\prime \prime}$ which vanishes only at the points of $\mathcal{R}$ and intersects the zero section transversely. Then the sections $s_{0}$ and $\bar{s}_{0}$ (defined from $s_{0}$ as above) endow $K^{\prime \prime}$ with a structure of a quaternionic line bundle over the complement of $\mathcal{R}$. Dividing $s_{0}$ by the square of its (quaternionic) norm produces a nowhere vanishing section $s$ of $K^{\prime \prime}$ over the complement of $\mathcal{R}$ and this section $s$ induces the required bundle morphism $\mathbf{F}^{\prime \prime}$.

Note that close to any $\left[A_{k}, 0\right] \in \mathcal{R}$, the norms of linear maps $\mathbf{F}_{[A, 0]}^{\prime \prime}$ are bounded below by some positive constant times the distance from $[A, 0]$ to $\left[A_{k}, 0\right]$. 
The morphisms $\mathbf{F}^{\prime}$ and $\mathbf{F}^{\prime \prime}$ together define a $J$-equivariant morphism $\mathbf{F}: K \rightarrow$ $T^{*} \times \mathbf{H}^{n}$ which is injective on all the fibres except over the points of $\mathcal{R}$ where the kernels can be identified with a copy of $\mathbf{H}$. We think of the pair $(\overline{\boldsymbol{D}}, \mathbf{F})$ as the family of Dirac operators associated to the stabilized Seiberg-Witten equations (which are defined below). Note that by construction of $\overline{\boldsymbol{D}}$ and $\mathbf{F}$, the associated family of Dirac operators has nontrivial kernels only at the points of $\mathcal{R}$, thus proving the last statement of the proposition.

To finish the construction of the stabilized equations, we need to globalize the perturbation terms $\mathbf{G}$ and $\mathbf{F}$. Let $P: \mathcal{K} \rightarrow \widetilde{T}^{*}$ be the $L^{2}$-orthogonal projection (where we treat $A_{0}$ as the origin of the above affine spaces), $Q: \mathcal{K} \rightarrow\left(\widetilde{T}^{*}\right)^{\perp}$ the orthogonal projection to the complement, and $\Pi: \boldsymbol{\Gamma}\left(W^{+}\right) \oplus T^{*} \times \mathbf{H}^{n} \rightarrow K$ the orthogonal projection to the kernel of $\overline{\boldsymbol{D}}$. The morphism $\mathbf{F}$ defines a map

$$
F: \mathcal{K} \oplus \Gamma\left(W^{+}\right) \oplus \mathbf{H}^{n} \rightarrow \mathbf{H}^{n},
$$

given by $F(A, \psi, w)=p r_{2} \circ \mathbf{F}(\Pi([P(A), \psi, w]))$. Similarly, $\mathbf{G}$ gives rise to

$$
G: \mathcal{K} \oplus \Gamma\left(W^{+}\right) \oplus \mathbf{H}^{n} \rightarrow \Gamma\left(W^{-}\right),
$$

which is well defined up to gauge change by $[P(A), G(A, \psi, w)]=\mathbf{G}([P(A), 0], w)$; it is completely determined by the appropriate choice of $G_{A_{0}}$.

We define the stabilized Seiberg-Witten equations via a map

$$
\overline{S W}: \mathcal{K} \oplus \Gamma\left(W^{+}\right) \oplus \mathbf{H}^{n} \rightarrow i \Omega_{+}^{2}(X) \oplus \Gamma\left(W^{-}\right) \oplus \mathbf{H}^{n},
$$

which is the sum of the original Seiberg-Witten map and the stabilization term given by

$$
\begin{aligned}
&(A, \psi, w) \mapsto \beta(Q(A), \psi, w) \cdot(0, G(A, \psi, w), F(A, \psi, w))+ \\
&(1-\beta(Q(A), \psi, w)) \cdot(0,0, w),
\end{aligned}
$$

where $\beta$ depends smoothly on the $L_{2}^{2}$-norms of $A$ and $\psi$ and on the norm of $w$ in such a way that it is equal to 1 in a small neighborhood of $(0,0,0)$ and equal to 0 in a slightly bigger neighborhood; notice that $\beta(Q(-),-,-)$ is invariant under the action of the gauge group $\mathcal{G}_{0}$ as well as under the action of $J$. It is clear from the nature of the perturbation terms that the moduli space of solutions to the stabilized Seiberg-Witten equation $\overline{S W}=0$ still contains the torus of reducibles $T^{*}$. This proves the proposition.

Remark. The proof of the proposition implies not only that $T^{*}$ is contained in the moduli space of solutions to $\overline{S W}=0$, but also that it is isolated, at least away from the points of $\mathcal{R}$. More precisely, for any neighborhood $U$ of $\mathcal{R}$, the complement $T^{*} \backslash U$ is isolated in the moduli space. This follows from the fact that $\mathbf{F}$ is injective on the kernels of the perturbed family of Dirac operators $\overline{\boldsymbol{D}}$ (along $T^{*}$ ) away from $\mathcal{R}$. 


\section{Kuranishi maps at the points of $\mathcal{R}$}

In this section we will construct a further perturbation of the stabilized Seiberg-Witten map $\overline{S W}$ whose solution space has a particularly simple form in a neighborhood of $T^{*}$, as described in the proposition below. The perturbation is supported in a neighborhood of the set $\mathcal{R} \subset T^{*}$ and is constructed by modifying the Kuranishi maps at the points of $\mathcal{R}$; these are the only points on $T^{*}$ at which the stabilized Dirac operators have nontrivial kernels.

Proposition 3.1. There exists a $J \times \mathcal{G}_{0}$-equivariant perturbation of the stabilized Seiberg-Witten equations, such that the perturbed map $\overline{\overline{S W}}$ satisfies the following:

1. The torus of reducibles $T^{*}$ is contained and isolated in the moduli space of solutions to the perturbed equations $\overline{\overline{S W}}=0$.

2. Given a small generic $\omega \in i \Omega_{+}^{2}(X)$ there exists an invariant neighborhood $\mathcal{U}$ of $\widetilde{T}^{*}$, such that all the solutions to $\overline{\overline{S W}}=(\omega, 0,0)$ that lie in $\mathcal{U}$ are smooth and irreducible. More precisely, every point in $\mathcal{R}$ gives rise to a smooth circle of solutions to $\overline{\overline{S W}}=(\omega, 0,0)$ in $\mathcal{U}$, contributing \pm 1 to the invariant, and there are no other solutions in $\mathcal{U}$.

Proof. Points of $\mathcal{R}$ fall into two categories depending on whether they are $j$-fixed or not. We consider the former case first, making use of the $j$-equivariance of the Kuranishi map. Then we modify the argument to deal with the rest of the points in $\mathcal{R}$.

Suppose $\left[A_{k}, 0\right] \in \mathcal{R}$ is $j$-fixed. The Kuranishi model for the solutions to $\overline{S W}=0$ around $\left(A_{k}, 0,0\right)$ is given by a $J$-equivariant map $Q: \mathbf{R}^{4} \oplus \mathbf{H} \rightarrow \mathbf{R}^{3} \oplus \mathbf{H}$, where $\mathbf{R}^{4}$ corresponds to the harmonic 1 -forms, $\mathbf{R}^{3}$ to the self-dual harmonic 2 forms, and the quaternions represent the kernel and the cokernel of the perturbed Dirac operator at $A_{k}$. Note that the leading term of $Q$ is a quadratic polynomial map which we will make non-degenerate by a perturbation. Denote by $\bar{Q}_{1}, \bar{Q}_{2}$ the quadratic parts of the components of $Q$. In principle these maps from $\mathbf{R}^{4} \oplus \mathbf{H}$ can contain three sorts of terms: quadratic in the first or the second variable, or bilinear. Which terms really appear is determined by the $J$-equivariance. Recall that $j$ acts on $\mathbf{H}$ by right quaternionic multiplication and on the spaces of forms by multiplication by -1 , whereas $U(1)$ acts by complex multiplication on $\mathbf{H}$ and trivially on the spaces of forms. This forces $\bar{Q}_{1}$ to be quadratic in the second (quaternionic) variable and $\bar{Q}_{2}$ to be bilinear. Note that $j$-equivariance imposes extra restrictions on these terms; clearly $\bar{Q}_{1} \circ j=-\bar{Q}_{1}$. The second component satisfies $\bar{Q}_{2} \circ j=-j \circ \bar{Q}_{2}$ if we think of $\bar{Q}_{2}$ as a linear map $\mathbf{R}^{4} \rightarrow \operatorname{End}_{\mathbf{C}}(\mathbf{H})$.

We choose a non-degenerate $J$-invariant quadratic map $R_{k}: \mathbf{H} \rightarrow \mathbf{R}^{3}$ (with the associated linear map an isomorphism, cf. [12]) to perturb $\bar{Q}_{1}$. For all but finitely many $\tau$, the map $\bar{Q}_{1}+\tau R_{k}$ is non-degenerate in the above sense. Admissible perturbations of $\bar{Q}_{2}$ are of the form $(a, w) \mapsto L(a) w$, where $L(a)$ is a $\mathbf{C}$-linear map which anti-commutes with the $j$-action. The space $\mathcal{I}$ of such maps is 4-dimensional over $\mathbf{R}$ and its non-zero elements are isomorphisms. We choose the map $L_{k}: \mathbf{R}^{4} \rightarrow \mathcal{I}, a \mapsto L_{k}(a)$ to be an isomorphism. Then for almost all $\tau$ 
the map $\bar{Q}_{2}+\tau L_{k}$, where we interpret $\bar{Q}_{2}$ as a linear map $\mathbf{R}^{4} \rightarrow \mathcal{I}$, is an isomorphism. Notice that $\bar{Q}_{2}(a,-)$ is itself an isomorphism for $a \neq 0$; this follows from the construction of the linear perturbation $\mathbf{F}$. Moreover, the norms of these linear maps are bounded from below by $C\|a\|$ for some positive $C$. This means that we can choose $\tau$ small enough so that for $a \neq 0$ the perturbation term is dominated by the original (quadratic) map. The benefits of this perturbation are twofold; firstly, the only solutions to the perturbed equations close to $\left(A_{k}, 0,0\right)$ are the reducible ones. Secondly, for a generic $h \in \mathcal{R}^{3}$, the preimage of $(h, 0,0)$ under the perturbed Kuranishi map consists of exactly one circle of solutions, hence the point $\left(A_{k}, 0,0\right)$ contributes \pm 1 to the Seiberg-Witten invariant.

Consider now a point $\left(A_{k}, 0,0\right)$ with $\left[A_{k}, 0\right] \in \mathcal{R}$ not $j$-fixed. Such a point has its $j$-image in $\mathcal{R}$; to make the perturbation term $j$-equivariant in this case, we construct a $U(1)$-equivariant perturbation at $\left(A_{k}, 0,0\right)$ and use the $j$-action to define the perturbation at its $j$-image. Given only $U(1)$-equivariance for $\bar{Q}_{1}$ and $\bar{Q}_{2}$ in this case, the structure of these quadratic maps is not so restricted. Using additional properties of the Kuranishi map, we still conclude that $\bar{Q}_{1}$ is quadratic in the second variable and $\bar{Q}_{2}$ is bilinear. However, the space of $U(1)$-invariant quadratic polynomials $\mathbf{H} \rightarrow \mathbf{R}$ is four dimensional and $\bar{Q}_{2}(a,-)$ can be any $\mathbf{C}$ linear map, so there is no canonical choice of a good perturbation. To gain the same control over the solution space as for $j$-fixed points, we endow the kernel and the cokernel with a quaternionic structure. The perturbation terms can then be constructed as above, using right multiplication by the quaternion $j$ in place of the $j$-action. For the perturbation term $R_{k}: \mathbf{H} \rightarrow \mathbf{R}^{3}$, the associated linear map is surjective and for all but finitely many $\tau$, the map $\bar{Q}_{1}+\tau R_{k}$ is an epimorphism in the above sense. The perturbation of the second component gives rise to an injective map $a \mapsto L_{k}(a)$; again, for all but finitely many $\tau$ the map $a \mapsto$ $\bar{Q}_{2}(a,-)+\tau L_{k}(a)$ is a monomorphism. The remark about domination of the pertubation term $\tau L_{k}(a)$ by $\bar{Q}_{2}(a,-)$ holds as above, and so do the conclusions about the solution space.

We fix a small, generic $\tau$ and define the perturbation term as a sum of terms localized near the points of $\mathcal{R}$. For a point $\left[A_{k}, 0\right] \in \mathcal{R}$ define the perturbing map by

$$
(A, \psi, w) \mapsto \tau \beta_{k}(A, \psi, w) \cdot\left(R_{k}\left(\Pi_{k}(\psi, w)\right), L_{k}(P(A)) \Pi_{k}(\psi, w), 0\right),
$$

where $\Pi_{k}: \Gamma\left(W^{+}\right) \oplus \mathbf{H}^{n} \rightarrow K_{A_{k}}^{\prime \prime}=\mathbf{H}$ is the $L^{2}$-orthogonal projection and $\beta_{k}$ is a $[0,1]$-valued function depending smoothly on the norms of the arguments (using $A_{k} \equiv 0$ ), that has support inside a small neighborhood of $\left(A_{k}, 0,0\right)$ (the projection of which by $\left(P, \Pi_{k}\right)$ is contained in the domain of the Kuranishi map) and is equal to 1 on a smaller neighborhood. The moduli space of solutions to the perturbed equations $\overline{\overline{S W}}=0$ still contains the torus of reducibles $T^{*}$ and it is clear from above that this torus is isolated. 


\section{Completion of the argument}

First we observe, following the line of argument in [12], that the moduli space of solutions to the perturbed equations $\overline{\overline{S W}}=0$ is compact. Moreover, because the perturbed equations we use can be connected to the unperturbed equations by a 1-parameter family, the count of solutions we obtain coincides with the Seiberg-Witten invariant. In the complement of $\widetilde{T}^{*}$, the action of $J$ is free, and so we can choose a small $J$-equivariant perturbation with support away from $\widetilde{T}^{*}$ such that the corresponding moduli space is smooth away from $\widetilde{T}^{*}$. (This fits into the general scheme laid down in $\S 4.3 .6$ of [2] because the perturbation is simply a small Fredholm section of a bundle over $\left(\mathcal{C}^{\prime}-\widetilde{T}^{*}\right) / J$, pulled back to $\mathcal{C}^{\prime}$.) Because $j$ acts freely, the solutions in the complement of $\widetilde{T}^{*}$ are paired up, and this part of the moduli space contributes an even number to the Seiberg-Witten invariant.

Along the space of reducible solutions we proceed by choosing a small generic self-dual 2-form $\omega$ which has a nonzero harmonic projection. If $\omega$ is small enough, the solutions to $\overline{\overline{S W}}=(\omega, 0,0)$ in an invariant neighborhood $\mathcal{U}$ of $\widetilde{T}^{*}$ are described as follows. For every point in $\mathcal{R}$, there is a circle of solutions corresponding to the $U(1)$ orbit. There are $r$ such circles, each of which contributes \pm 1 to the invariant. All the rest of the solutions are paired by the $j$ action, hence the statement of the theorem follows.

\section{Some homology tori}

There are a number of examples of homology tori whose Seiberg-Witten invariants one can compute directly; it is interesting to see how these are consistent with our theorem. The simplest are the torus $T^{4}$, whose Seiberg-Witten invariant is \pm 1 , and the connected sum

$$
\#_{4} S^{1} \times S^{3} \# \#{ }_{3} S^{2} \times S^{2},
$$

whose Seiberg-Witten invariant vanishes. These manifolds have determinant 1 and 0 , respectively.

A more interesting class of examples is the set of manifolds of the form $X=$ $S^{1} \times M^{3}$, where $M$ is an orientable 3 -manifold with the homology of a torus. Work of Meng and Taubes shows how to compute the invariant of $X$, in terms of the Alexander polynomial of $M$. There are two parts to the computation. First, there is an identification of the Seiberg-Witten invariant of $X$ with the 3-dimensional Seiberg-Witten invariant of $M^{3}$. This is proved by a variant of the argument proving proposition 5.1 of [11]. In particular, the $\mathrm{Spin}^{\mathrm{c}}$ structures on $X$ with non-vanishing Seiberg-Witten invariant all pull back from $M$. The main theorem of [10] shows that the Seiberg-Witten invariant of $M$ (and therefore of $X$ ) has for generating function the multivariable Alexander polynomial of $M$. In light of Theorem $\mathrm{A}$, we explain how the determinant of $X$ is related to the Alexander polynomial of $M$. 
We define the determinant $\operatorname{det}(M)$ analogously to that of $X$, using the 3-fold cup product in $H^{1}(M)$. Note that the determinant of $S^{1} \times M$ coincides with that of $M$. The Alexander polynomial of $M, \Delta_{M}$, is a Laurent polynomial in variables $t_{1}^{ \pm 1}, t_{2}^{ \pm 1}, t_{3}^{ \pm 1}$ which is defined up to multiplication by $\pm t_{i}$. The relation we need is the following:

Lemma 5.1. If $M$ is a homology torus, then

$$
\Delta_{M}(1,1,1)= \pm \operatorname{det}(M)^{2}
$$

The Lemma may be deduced from work of L. Traldi [14] and J. Levine [6]. Those authors treat the Alexander polynomial $\Delta_{L}$ of an $n$-component link $L$ in a homology sphere; in our situation the homology sphere is obtained by doing surgery on a set of circles representing a basis of $H_{1}(M)$ and $L$ consists of the meridians of those circles. If the linking numbers between the components are all 0 , as is the case for us, they show that

$$
\frac{\Delta_{L}}{\left(t_{1}-1\right) \cdots\left(t_{n}-1\right)}=d_{0}+\text { higher order terms in } t_{i}-1
$$

where $d_{0}$ may be evaluated as a determinant involving the $\bar{\mu}$-invariants of $L$. (Compare [6, Corollary 1.6] and the proof of [14, Theorem 5.3].) When there are only 3 components, the determinant works out to be $\bar{\mu}_{123}(L)^{2}$. Now the quotient on the left-hand side of equation (1) is the Alexander polynomial of $M$, and it has been known for a long time [9] that the invariant $\bar{\mu}_{123}(L)$ coincides with the 3-fold Massey product.

In terms of Seiberg-Witten theory, the evaluation $\Delta_{M}(1,1,1)$ is the sum of the Seiberg-Witten invariants of all of the $\operatorname{Spin}^{\mathrm{c}}$ structures on $M$. Recall that there is an involution on the set of $\operatorname{Spin}^{\mathrm{c}}$ structures, whose only fixed point is the Spin ${ }^{\mathrm{c}}$ structure $S_{0}$ with trivial determinant, i.e. the one we have been studying. Hence we have the chain of equalities and congruences

$$
\mathrm{SW}_{X}\left(S_{0}\right)=\mathrm{SW}_{M}\left(S_{0}\right) \equiv \Delta_{M}(1,1,1)=\operatorname{det}(M)^{2} \equiv \operatorname{det}(M) \quad(\bmod 2),
$$

which is consistent with our main theorem since $\operatorname{det}(M)=\operatorname{det}(X)$.

It is not hard to find 3-manifolds with arbitrary determinant $\operatorname{det}(M)$; a simple construction is to take 0 -framed surgery on the $n$-fold band sum of the Borromean rings. The case $n=2$ is illustrated below in Figure 1. If each copy of the Borromean rings is oriented so that the triple Massey product is +1 , then $\operatorname{det}(M)=n$. 


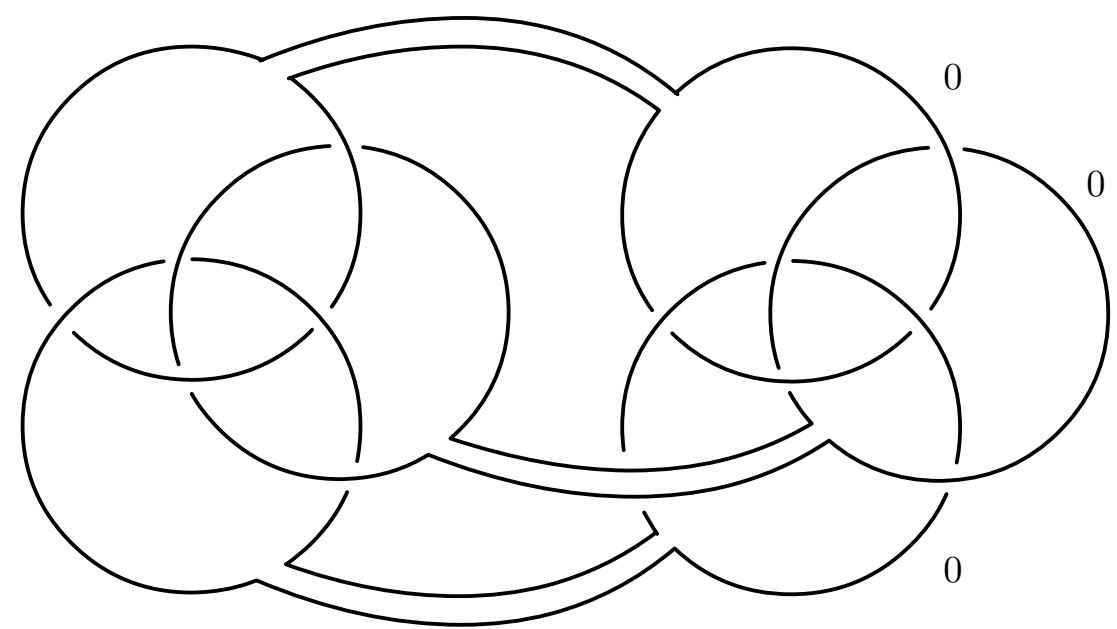

Figure 1

Remark. The calculations assembled above give rise to a curious criterion for a homology torus $X^{4}$ to be diffeomorphic to the product of $S^{1}$ and a 3-manifold. Namely, the sum of its Seiberg-Witten invariants should be a square (up to sign). It would be of interest to find an example where this criterion does not hold, but where $X$ is homeomorphic (or perhaps homotopy equivalent) to a product.

One last class of examples is obtained via the 'knot-surgery' construction of Fintushel and Stern. Following [3], let $K$ be a knot in $S^{3}$, with exterior $E_{K}$. Remove a copy of $T^{2} \times D^{2}$ from $T^{4}$, and glue in $S^{1} \times E_{K}$, resulting in a new manifold $X_{K}$ with the same cohomology as $T^{4}$. It is not hard to see that $X_{K}$ is in fact $S^{1} \times M$, where $M$ is gotten by replacing a copy of $S^{1} \times D^{2} \subset T^{3}$ with $E_{K}$. From this, or from gluing theorems (cf. [3, Theorem 1.5]), it follows that the Seiberg-Witten invariant of $X_{K}$ is $\Delta_{K}\left(T^{2}\right)$. To make a manifold which is not a product, perform this construction on three disjoint tori $T_{1}, T_{2}, T_{3}$ (using knots $K_{1}, K_{2}, K_{3}$ ) in different (non-zero) homology classes, as in [4], to get a manifold $X_{K_{1}, K_{2}, K_{3}}$. Suppose that the knot-surgery is performed so that the circle factor in each $S^{1} \times E_{K_{i}}$ is glued to the same circle factor in $T^{4}$. The result is a product of $S^{1}$ with the manifold obtained by 0 -surgery on the Borromean rings with the knots $K_{i}$ tied in the three rings. If the circle factors in each $S^{1} \times E_{K_{i}}$ are glued to different circles in $T^{4}$ (and the knots are non-trivial) then $X_{K_{1}, K_{2}, K_{3}}$ cannot be written as $S^{1}$ times any 3 -manifold. This is verified by a fundamental group calculation; on the other hand the Seiberg-Witten invariants are independent of the gluing and are given by

$$
\Delta_{K_{1}}\left(T_{1}^{2}\right) \Delta_{K_{2}}\left(T_{2}^{2}\right) \Delta_{K_{3}}\left(T_{3}^{2}\right)
$$

\section{References}

[1] M. F. Atiyah, K-theory and reality, Quart. J. Math. Oxford Ser. 17 (1966), 367-386.

[2] S. Donaldson and P. Kronheimer, The Geometry of Four-Manifolds, Clarendon Press, Oxford, 1990. 
[3] R. Fintushel and R. Stern, Knots, links, and 4-manifolds, Invent. Math. 134 (1998), $363-400$.

[4] R. Gompf and T. Mrowka, Irreducible 4-manifolds need not be complex, Ann. of Math. 138 (1993), 61-111.

[5] K. Iriye, M. Mimura, K. Shimakawa, and M. Yasuo, A quaternionic analogue of Atiyah's real K-theory, Transformation group theory (Taejŏn, 1996), Korea Adv. Inst. Sci. Tech., Taejŏn, 51-61.

[6] J. Levine, A factorization of the Conway polynomial, Comment. Math. Helv., 74 (1999), $27-53$.

[7] J. Li and G. Tian, Virtual moduli cycles and Gromov-Witten invariants of algebraic varieties, J. Amer. Math. Soc. 11 (1998), 119-174.

[8] T. J. Li and A. Liu, General wall crossing formula, Math. Res. Lett. 2 (1995), 797-810.

[9] W. S. Massey, Higher order linking numbers, J. Knot Theory Ramifications 7 (1998), 393-414.

[10] G. Meng and C. H. Taubes, $\underline{\mathrm{SW}}=$ Milnor torsion, Math. Res. Lett. 3 (1996), 661-674.

[11] J. Morgan, Z. Szabó, and C. Taubes, A product formula for the Seiberg-Witten invariants and the generalized Thom conjecture, J. Differential Geom. 44 (1996), 706-788.

[12] J. W. Morgan and Z. Szabó, Homotopy K3 surfaces and mod 2 Seiberg-Witten invariants, Math. Res. Lett. 4 (1997), 17-21.

[13] D. Salamon, Spin Geometry and Seiberg-Witten Invariants, 1996. Birkhauser Verlag, to appear (2000).

[14] L. Traldi, Milnor's invariants and the completions of link modules, Trans. Amer. Math. Soc. 284 (1984), 401-424.

Department of Mathematics, MS 050, Brandeis University, Waltham MA 02454.

E-mail address: ruberman@brandeis.edu

E-mail address: strle@brandeis.edu 